\title{
Supplementary Information for Development and Application of a Novel Semi-quantification Approach in LC-QToF-MS Analysis of Natural Products
}

\author{
Reza Aalizadeh, Anthi Panara and Nikolaos S. Thomaidis* \\ Laboratory of Analytical Chemistry, Department of Chemistry, National and Kapodistrian \\ University of Athens, Panepistimiopolis Zografou, 15771 Athens, Greece
}

*Correspondence should be addressed to Prof. Nikolaos S. Thomaidis.

E-mail: ntho@chem.uoa.gr

Tel: +302107274317

Fax: +302107274750

Running title: LC-HRMS and semi-quantification in Natural Products

Keywords: LC-HRMS, semiquantification, chemometrics, natural products 
Figure S1 shows the effect of inclusion/exclusion of ethyl vanillin as internal standard in calculation of relative peak area and logIE values. In quantitative analysis, it is normal and routine to divide peak area of signal of analyte with an internal standard (IS) to remove variation in signal caused by instrumental sensitivity. Here we avoided to use relative peak area for majority of compounds because they were linear and $\log I \mathrm{E}$ values were not significantly different between pure and relative peak area. This further simplifies the derivation of predicted concentration from $\log \mathrm{IE}$ values. In the calculation of $\log \mathrm{IE}$, the slope of calibration curve of every reference standard is divided to the slope of calibration curve of "tyrosol", the logIE values are already relative ones and they are not absolute values. Below is the calculation for logIE values with and without internal standard is exemplified for "Salicylic acid" (without influential analysis for linearity check) under $\mathrm{ACN}: \mathrm{H}_{2} \mathrm{O}$ gradient with and without IS ("ethyl vanillin"):

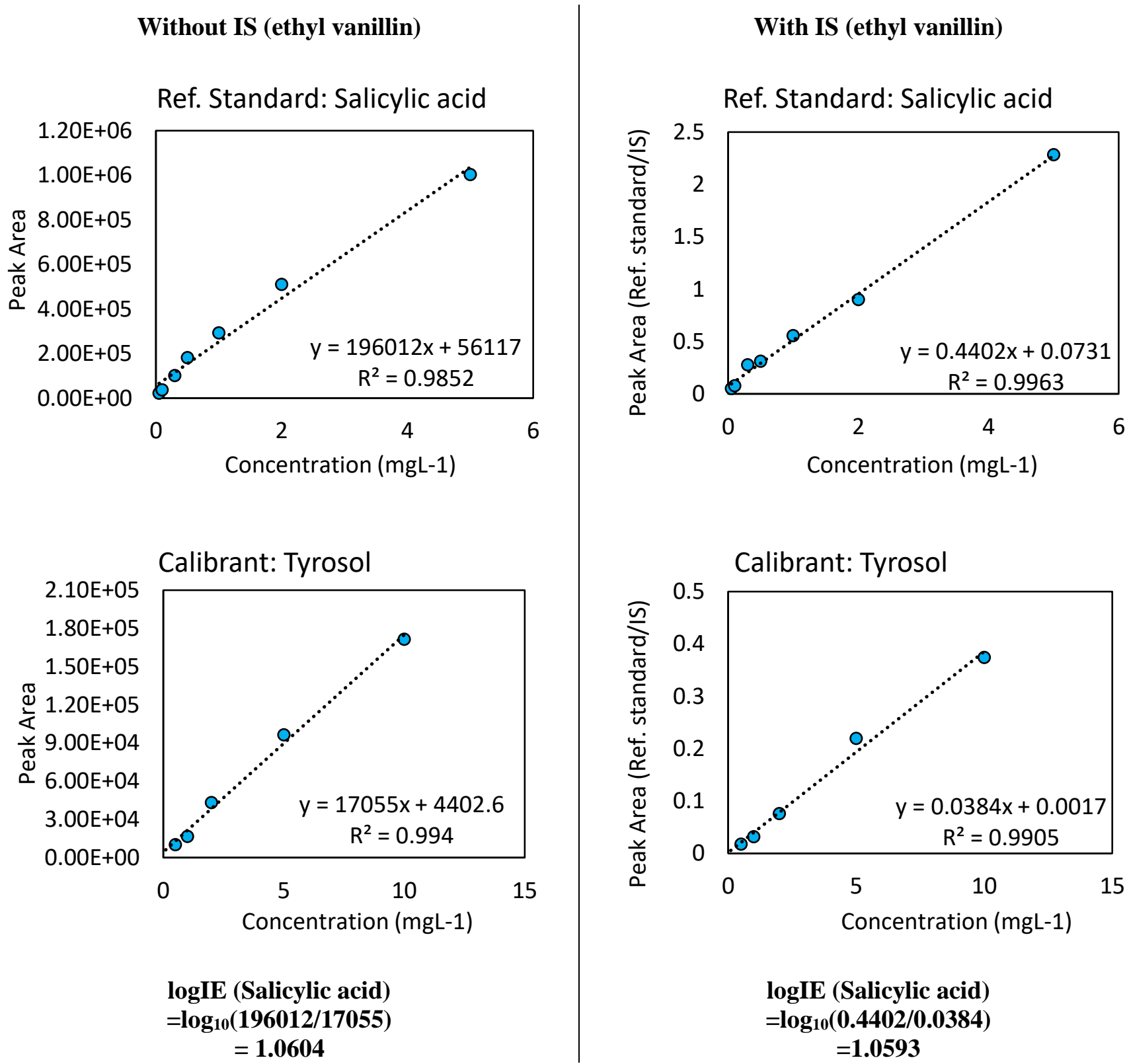

Figure S1. Variation in logIE values when pure/relative peak area was used 
As it can be seen the difference between logIE values that constructed with and without inclusion of IS in slopes is $\mathbf{\pm 0 . 0 0 1 1}$. This is not statistically significant as Monte carlo Sampling approach provided the maximum accepted error of 0.605 at $95 \%$ quantile which means that this error would affect the predictions. 